\title{
Assessment of an ${ }^{18}$ F-Labeled Phosphoramidate Peptidomimetic as a New Prostate-Specific Membrane Antigen-Targeted Imaging Agent for Prostate Cancer
}

Suzanne E. Lapi ${ }^{1}$, Hilla Wahnishe ${ }^{1}$, David Pham ${ }^{1}$, Lisa Y. Wu², Jessie R. Nedrow-Byers ${ }^{2}$, Tiancheng Liu ${ }^{2}$, Kaveh Vejdani ${ }^{1}$, Henry F. VanBrocklin*1, Clifford E. Berkman*2,3, and Ella F. Jones*1

${ }^{1}$ Department of Radiology and Biomedical Imaging, Center for Molecular and Functional Imaging, University of California, San Francisco, California; ${ }^{2}$ Department of Chemistry, Washington State University, Pullman, Washington; and ${ }^{3}$ Cancer Targeted Technology, LLC, Woodinville, WA

Prostate-specific membrane antigen (PSMA) is a transmembrane protein commonly found on the surface of late-stage and metastatic prostate cancer and a well-known imaging biomarker for staging and monitoring therapy. Although ${ }^{111}$ In-labeled capropmab pendetide is the only approved agent available for PSMA imaging, its clinical use is limited because of its slow distribution and clearance that leads to challenging image interpretation. A small-molecule approach using radiolabeled urea-based PSMA inhibitors as imaging agents has shown promise for prostate cancer imaging. The motivation of this work is to explore phosphoramidates as a new class of potent PSMA inhibitors to develop more effective prostate cancer imaging agents with improved specificity and clearance properties. Methods: $\mathrm{N}$-succinimidyl-4- ${ }^{18} \mathrm{~F}$-fluorobenzoate ( $\left.{ }^{18} \mathrm{~F}-\mathrm{SFB}\right)$ was conjugated to $S$-2-((2-(S-4-amino-4-carboxybutanamido)-S-2-carboxyethoxy)hydroxyphosphorylamino)-pentanedioic acid (Phosphoramidate (1)), yielding $S-2-((2-(S-4-(4-18$ F-fluorobenzamido)-4-carboxybutanamido)-S-2-carboxyethoxy)hydroxyphosphorylamino)pentanedioic acid (3). In vivo studies were conducted in mice bearing either LNCaP (PSMA-positive) or PC-3 (PSMA-negative) tumors. PET images were acquired at 1 and $2 \mathrm{~h}$ with or without a preinjection of a nonradioactive version of the fluorophosphoramidate. Tissue distribution studies were performed at the end of the $2 \mathrm{~h}$ imaging sessions. Results: Phosphoramidate (1) and its fluorobenzamido conjugate (2) were potent inhibitors of PSMA (inhibitory concentration of $50 \%$ [ $\left.\mathrm{IC}_{50}\right], 14$ and $0.68 \mathrm{nM}$, respectively). PSMA-mediated tumor accumulation was noted in the LNCaP versus the PC-3 tumor xenografts. The LNCaP tumor uptake was also blocked by the administration of nonradioactive (2) prior to imaging studies. With the exception of the kidneys, tumor-to-tissue and tumor-to-blood ratios were greater than 5:1 at $2 \mathrm{~h}$. The strong kidney uptake may be due to the known PSMA expression in the mouse kidney, because significant reduction ( $>6$-fold) in kidney activity was seen in mice

Received May 26, 2009; revision accepted Aug. 28, 2009.

For correspondence or reprints contact: Ella F. Jones, Department of Radiology and Biomedical Imaging, Center for Molecular and Functional Imaging, University of California, San Francisco, 185 Berry St., Suite 350, Box 0946, San Francisco, CA 94107.

E-mail: ella.jones@radiology.ucsf.edu

${ }^{*}$ Contributed equally to this work.

COPYRIGHT @ 2009 by the Society of Nuclear Medicine, Inc. injected with (2). Conclusion: ${ }^{18} \mathrm{~F}$-labeled phosphoramidate (3) is a representative of a new class of PSMA targeting peptidomimetic molecules that shows great promise as imaging agents for detecting PSMA+ prostate tumors.

Key Words: molecular imaging; PET; radiopharmaceuticals; ${ }^{18} \mathrm{~F}$; PSMA; phosphoramidate; prostate cancer

J Nucl Med 2009; 50:2042-2048

DOI: 10.2967/jnumed.109.066589

$\mathbf{P}$ rostate cancer is the second leading cancer found in men. Each year in the United States, more than 186,000 new cases are diagnosed, with approximately 29,000 prostate cancer-related deaths (1). Prostate cancer imaging not only is an important component of diagnosis and staging, it has also become an integral part of treatment planning, especially in radiation therapy (2).

Prostate-specific membrane antigen (PSMA) is a 100 to $120 \mathrm{kDa}$ transmembrane protein upregulated on the tumor cell surface of late-stage, androgen-independent, and metastatic prostate cancer (3). Because of its restricted overexpression on prostate cancer cells, PSMA is an important biomarker for prostate cancer prognosis and an attractive target for therapy (4). ${ }^{111}$ In-labeled capropmab pendetide (ProstaScint; Cytogen) is an antibody-based agent approved by the Food and Drug Administration for PSMA imaging. Although it is the only commercially available agent, its clinical use is limited largely because of its slow distribution and clearance, and the images produced are often difficult to interpret. More recently, a small-molecule approach has generated a class of promising urea-based PSMA-targeted agents (Fig. 1) for PET and SPECT (5-7). Although optimization of these tracers is under way, exploration of more potent PSMA-targeting groups and their use for prostate cancer imaging will produce new imaging agents with improved sensitivity and specificity. 


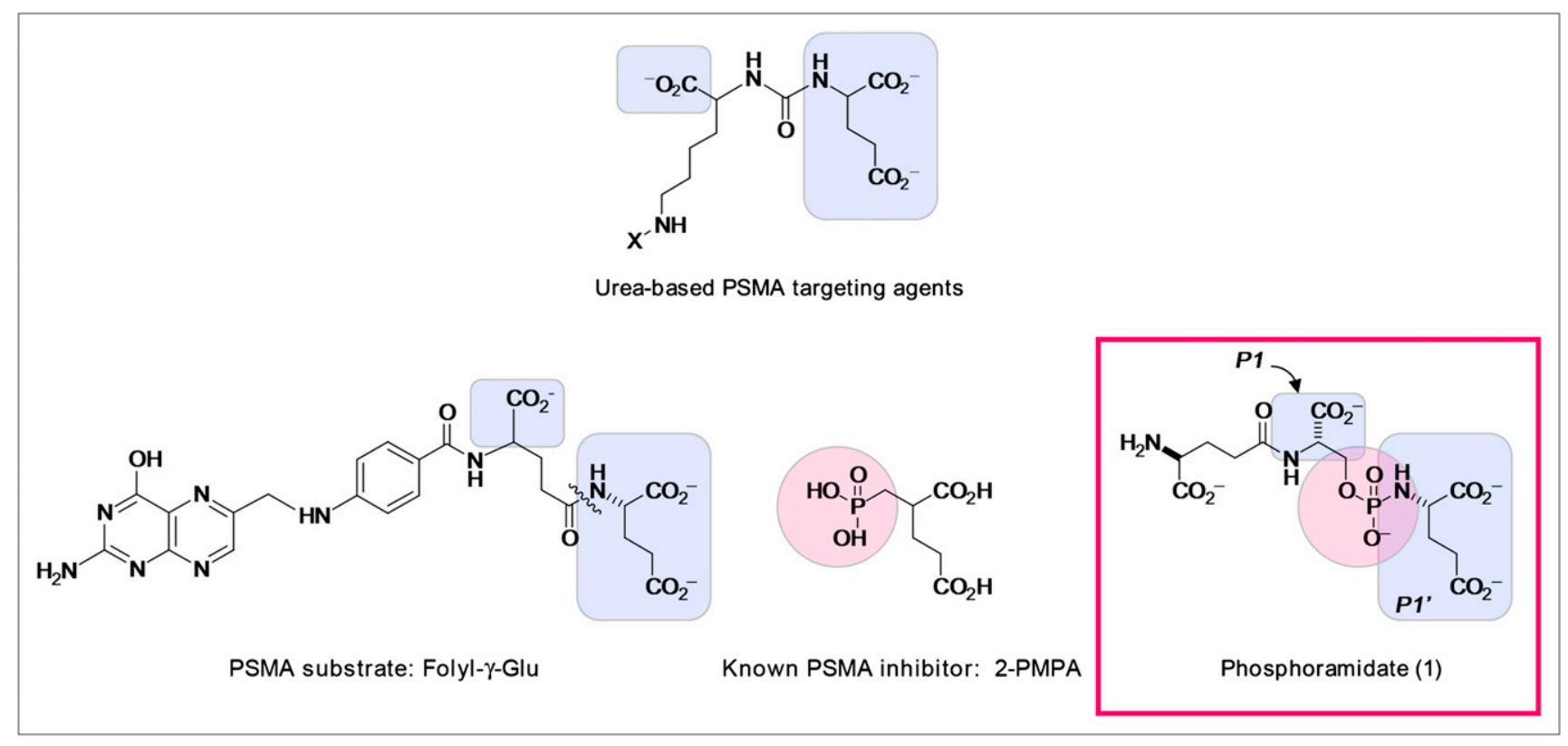

FIGURE 1. Structural elements of known PSMA substrate and inhibitors, compared with phosphoramidate (1). Highlighted portions indicate structural features similar to phosphoramidate design.

Also known as folate hydrolase I and glutamate carboxypeptidase II, PSMA is reported to possess proteolytic activities toward $\gamma$-glutamyl folic acid derivatives (Fig. 1) and the neuropeptide $N$-acetylaspartylglutamate $(8,9)$. Further studies on the PSMA substrate specificity have indicated that acidic residues at the $\mathrm{P} 1$ and $\mathrm{P}^{\prime}$ positions are more preferable, and several folate-like and $\mathrm{N}$-acetylaspartylglutamatelike dipeptides with modest hydrolytic efficiency have been identified (10). In our own work, while adapting the dipeptide motif for PSMA targeting, we have developed a library of tetrahedral phosphoramidates for PSMA inhibition (11). Through molecular pruning, we have systematically identified several potent inhibitors (12) that pseudoirreversibly bind to PSMA (13). In vitro studies of the fluorescently labeled phosphoramidates further reveal their ability to localize and internalize in PSMA-positive (PSMA+) cells (14), making these compounds ideal candidates for PSMA-targeted delivery for prostate cancer imaging and therapeutic applications.

Herein we present our effort in using the phosphoramidate scaffold as a targeting element for prostate cancer imaging. The synthesis and characterization of an ${ }^{18} \mathrm{~F}-$ labeled analog of phosphoramidate (1) and its in vivo PET and biodistribution data in murine xenografts are reported.

\section{MATERIALS AND METHODS}

\section{Cell Lines, Reagents, and General Methods}

LNCaP and PC-3 cells were obtained from the American Type Culture Collection. ${ }^{1} \mathrm{H},{ }^{13} \mathrm{C}$, and ${ }^{31} \mathrm{P}$ nuclear magnetic resonance (NMR) spectra were recorded on a 300- and 500-MHz (DRX; Bruker) or a $400-\mathrm{MHz}$ (Varian) NMR spectrometer. ${ }^{1} \mathrm{H}$ NMR chemical shifts are relative to tetramethylsilane $(\delta=0.00$ parts per million $[\mathrm{ppm}]), \mathrm{CDCl}_{3}(\delta=7.26 \mathrm{ppm})$, or $\mathrm{D}_{2} \mathrm{O}(\delta=4.87$ ppm). ${ }^{13} \mathrm{C}$ NMR chemical shifts are relative to $\mathrm{CDCl}_{3}(\delta=77.23$ ppm). ${ }^{31} \mathrm{P}$ NMR chemical shifts in $\mathrm{CDCl}_{3}$ or $\mathrm{D}_{2} \mathrm{O}$ were externally referenced to $85 \% \mathrm{H}_{3} \mathrm{PO}_{4}(\delta=0.00 \mathrm{ppm})$ in $\mathrm{CDCl}_{3}$ and $\mathrm{D}_{2} \mathrm{O}$, respectively. Aqueous solutions were prepared with deionized distilled water (Milli-Q water system; Millipore). All liquid flash chromatography (silica or C18) was performed using a Biotage SP4 flash chromatography system. The high-performance liquid chromatography (HPLC) analysis and purification system for radioactive compounds consisted of a Rheodyne injector with a 2-mL loop, a Waters model 590 pump, a Shimudzu model SPD$10 \mathrm{~A}$ ultraviolet detector, and an in-line radioactivity detector (model $105 \mathrm{~s}$; Carroll and Ramsey Associates) coupled to a data collection system (PeakSimple, model 304; SRI). The phosphoramidate precursor 2-benzyloxycarbonylamino-4-(1-benzyloxycarbonyl-2-hydroxy-ethylcarbamoyl)-butyric acid benzyl ester was synthesized as previously described (14). $t$-Butyl $4-N, N, N$, trimethyl ammonium benzoate triflate salt was synthesized according to the literature method (15). $N$-succinimidyl-4-fluorobenzoate (SFB) was prepared as previously described (16). All other reagents and solvents were purchased from Sigma-Aldrich and Fisher Scientific and were used without further purification unless indicated.

\section{Synthesis of S-2-((2-)(S-4-Amino-4-}

Carboxybutanamido)-S-2-Carboxyethoxy)-

Hydroxyphosphorylamino)-Pentanedioic Acid (1)

A tetrahydrofuran solution $(15 \mathrm{~mL})$ of 2-benzyloxycarbonylamino-4-(1-benzyloxycarbonyl-2-hydroxy-ethylcarbamoyl)-butyric acid benzyl ester (71 mg, $0.069 \mathrm{mmol}$ ) was added with $10 \% \mathrm{Pd} / \mathrm{C}$ (12 mg), $\mathrm{K}_{2} \mathrm{CO}_{3}$ (23 mg, 2 equivalent), and distilled $\mathrm{H}_{2} \mathrm{O}$ (1 mL). The mixture was stirred vigorously under $\operatorname{Ar}(\mathrm{g})$, followed by the charge of $\mathrm{H}_{2}(\mathrm{~g})$ from a balloon for $7 \mathrm{~h}$ at room temperature. After the reaction was completed, the solvent was removed under vacuum. The remaining residue was dissolved in 1:1 ratio of 
methanol/water, filtered through a 0.2-mm PTFE micropore filtration disk (Whatman), and dried under vacuum. The yield was $89.9 \% .{ }^{1} \mathrm{H}$ NMR $\left(300 \mathrm{MHz}, \mathrm{D}_{2} \mathrm{O}\right): \delta 1.75-1.94(\mathrm{~m}, 2 \mathrm{H})$, 1.98-2.10 (m, 2H), 2.12-2.20 (m, 2H), 2.43-2.51 (m, 2H), 3.47$3.54(\mathrm{q}, 1 \mathrm{H}, \mathrm{J}=6 \mathrm{~Hz}, 15 \mathrm{~Hz}), 3.63-3.67(\mathrm{q}, 1 \mathrm{H}, \mathrm{J}=5 \mathrm{~Hz}, 8 \mathrm{~Hz})$, $3.98-4.10(\mathrm{~m}, 2 \mathrm{H})$, and $4.32-4.35(\mathrm{t}, 1 \mathrm{H}, \mathrm{J}=4 \mathrm{~Hz}) .{ }^{13} \mathrm{C}$ NMR $(75$ $\left.\mathrm{MHz}, \mathrm{D}_{2} \mathrm{O}\right): \delta$ 26.8, 31.4, 31.7, 31.8, 33.7, 48.7, 54.2, 55.6, 55.8, 56.6, 64.6, 64.7, 174.3, 175.21, 176.0, 181.5, 181.6, and 182.9. ${ }^{31} \mathrm{P}$ NMR $\left(300 \mathrm{MHz}, \mathrm{D}_{2} \mathrm{O}\right): \delta 7.63$.

\section{Synthesis of S-2-((2-(S-4-(4-Fluorobenzamido)-}

4-Carboxybutanamido)-S-2-Carboxyethoxy)

Hydroxyphosphorylamino)-Pentanedioic Acid (2)

A solution of $N$-succinimidyl-4-fluorobenzoate (SFB) $(6 \mu \mathrm{mol})$ in $100 \mu \mathrm{L}$ of DMSO was added to a stirred solution of (1) ( $2 \mu \mathrm{mol}, 100 \mu \mathrm{L}$ of $20 \mathrm{mM}$ in $\mathrm{H}_{2} \mathrm{O}$ ), $160 \mu \mathrm{L}$ of $\mathrm{H}_{2} \mathrm{O}$, and $40 \mu \mathrm{L}$ of $1 \mathrm{M} \mathrm{NaHCO}_{3}$. The reaction mixture was stirred for $6 \mathrm{~h}$ in the dark at room temperature. The $\mathrm{pH}$ of the resulting solution was then adjusted to 9.3 by the addition of $8 \mu \mathrm{L}$ of $1 \mathrm{M} \mathrm{Na}_{2} \mathrm{CO}_{3}$. The unreacted (1) was scavenged by stirring with $25 \mathrm{mg}$ of the SiIsocyanate resin (SiliCycle, Inc.) overnight at room temperature. The solution was subsequently centrifuged $(7,800$ relative centrifugal force, $10 \mathrm{~min}$ ), and the supernatant was lyophilized in a 2-mL microcentrifuge tube. The unreacted or hydrolyzed SFB was removed by successively triturating the lyophilized solid with $1-\mathrm{mL}$ portions of DMSO and centrifuging the mixture $(16,200$ relative centrifugal force, $1 \mathrm{~min}$ ) after each wash; this process was repeated 10 times. The resulting solid was dried in vacuo providing the desired 4-fluorobenzamido-phosphoramidate (2) in quantitative yield. ${ }^{1} \mathrm{H}$ NMR $\left(\mathrm{D}_{2} \mathrm{O}\right)$ : d $1.56-1.68(\mathrm{~m}, 2 \mathrm{H}), 1.86-$ $2.14(\mathrm{~m}, 4 \mathrm{H}), 2.27-2.35(\mathrm{~m}, 2 \mathrm{H}), 3.27-3.34\left(\mathrm{~m},{ }^{1} \mathrm{H}\right), 3.73-3.87$ (m, 2H), 4.05-4.08 (t, $\left.{ }^{1} \mathrm{H}, 3.9 \mathrm{~Hz}\right), 4.15-4.19$ (dd, ${ }^{1} \mathrm{H}, 4.4 \mathrm{~Hz}, 9.15$ $\mathrm{Hz}$ ), 7.04-7.10 (t, 2H, 9.0 Hz, 9.0 Hz), and 7.66-7.70 (dd, 2H, 9.0 $\mathrm{Hz}, 5.3 \mathrm{~Hz}) .{ }^{13} \mathrm{C}$ NMR $\left(\mathrm{D}_{2} \mathrm{O}\right)$ : d 27.51, 32.04, 32.08, 32.69,33.95, $55.96,56.05,56.83,64.90,115.63,115.80,139.90,129.97$, $160.81,163.88,165.87,169.66,175.37,176.13,178.75,181.66$, and 183.19. ${ }^{31} \mathrm{P}$ NMR $\left(\mathrm{D}_{2} \mathrm{O}\right)$ : d 8.33. Matrix-assisted laser desorption ionization high-resolution mass spectrometry (M-K) ${ }^{-}$ calculated 715.9266, found 715.9359 for $\mathrm{C}_{20} \mathrm{H}_{20} \mathrm{FK}_{4} \mathrm{~N}_{3} \mathrm{O}_{10} \mathrm{P}$.

\section{Synthesis of $\mathbf{N}$-Succinimidyl-4-18 F-Fluorobenzoate $\left({ }^{18} \mathrm{~F}-\mathrm{SFB}\right)$}

${ }^{18} \mathrm{~F}-\mathrm{SFB}$ was synthesized based on the literature method with modifications (17). Briefly, ${ }^{18} \mathrm{~F}$-fluoride ion was produced by proton bombardment of ${ }^{18} \mathrm{O}-\mathrm{H}_{2} \mathrm{O}$ using the University of California, San Francisco (UCSF), PETtrace (GE Healthcare) 17$\mathrm{MeV}$ cyclotron. The ${ }^{18} \mathrm{~F}$-fluoride ion was concentrated on an anion exchange column (Oak Ridge Technology Group Inc.) and eluted with $0.5 \mathrm{~mL}$ (95\% acetonitrile/water) in a solution of Kryptofix $222(4,7,13,16,21,24$-hexaoxa-1,10-diazabicyclo[8.8.8]hexacosane- $\left.\mathrm{K}_{222} ; 30 \mathrm{mg} / \mathrm{mL}\right)$ and $\mathrm{K}_{2} \mathrm{CO}_{3}(5.5 \mathrm{mg} / \mathrm{mL})$. The water was removed azeotropically by the addition and evaporation of acetonitrile. A DMSO solution $(0.5 \mathrm{~mL})$ of $t$-butyl $4-N, N, N$, trimethyl ammonium benzoate triflate salt $(2 \mathrm{mg}, 11 \mu \mathrm{mol})$ was added and allowed to react at $160^{\circ} \mathrm{C}$ for $10 \mathrm{~min}$. After cooling, 0.6 $\mathrm{mL}$ of $1 \mathrm{M} \mathrm{HCl}$ was added, and the solution was heated to $120^{\circ} \mathrm{C}$ for $15 \mathrm{~min}$. The solution mixture was diluted to $10 \mathrm{~mL}$ with water and passed through a Chromafix HR-P cartridge (MachereyNagel). ${ }^{18} \mathrm{~F}$-fluorobenzoic acid was eluted from the cartridge with acetonitrile, treated with $20 \mu \mathrm{L}$ of methanolic $\left(\mathrm{CH}_{3}\right)_{4} \mathrm{NOH}$ in $0.4 \mathrm{~mL}$ of acetonitrile, and brought to dryness under a nitrogen stream. $O$-( $N$-succinimidyl)- $N, N, N^{\prime}, N^{\prime}$-tetramethyluroniumtetrafluoroborate $(10 \mathrm{mg}, 33 \mu \mathrm{mol})$ in $0.5 \mathrm{~mL}$ of acetonitrile was added to the resulting residue and allowed to react at $60^{\circ} \mathrm{C}$ for $5 \mathrm{~min}$. After cooling, the solution mixture was diluted with $0.5 \%$ acetic acid to $10 \mathrm{~mL}$ and loaded onto a second Chromafix HR-P cartridge. The cartridge was washed with $5 \mathrm{~mL}$ of $20 \%$ acetonitrile and eluted with $1.5 \mathrm{~mL}$ of acetonitrile. Radiochemical yields were typically approximately $20 \%$ decay-corrected from fluoride.

\section{Synthesis of ${ }^{18}$ F-S-2-((2-(S-4-(4-Fluorobenzamido)- 4-Carboxybutanamido)-S-2-Carboxyethoxy) Hydroxyphosphoryl-Amino)-Pentanedioic Acid (3)}

The synthesized ${ }^{18} \mathrm{~F}-\mathrm{SFB}$ was brought to dryness under reduced pressure with a nitrogen stream. It was dissolved in $50 \mu \mathrm{L}$ of acetonitrile and allowed to react with $3-4 \mathrm{mg}$ of phosphoramidate (1) (dissolved in $100 \mu \mathrm{L}$ of $\mathrm{H}_{2} \mathrm{O}$ ) and $100 \mu \mathrm{L}$ of sodium carbonate buffer $(1 \mathrm{M}), \mathrm{pH} 10$. The reaction was allowed to proceed for 10 $\mathrm{min}$ at room temperature, and the radioconjugate was purified by HPLC (Synergi Fusion-RP 80A column; Phenomenex) $(250 \mathrm{~mm} \times$ $4.6 \mathrm{~mm}, 4 \mu \mathrm{m}, 100 \mathrm{mM}$ sodium phosphate buffer, $\mathrm{pH} 6.8$, as eluant; flow rate, $1 \mathrm{~mL} / \mathrm{min}$; elution time, $5 \mathrm{~min}$ ). Radiochemical yields (from ${ }^{18} \mathrm{~F}-\mathrm{SFB}$ ) were typically greater than $90 \%$.

\section{General Method of Determining Inhibitory Concentration of $50 \%\left(\mathrm{IC}_{50}\right)$}

Inhibition studies were performed as previously described, with minor modifications $(12,14)$. Working solutions of the substrate ( $N$-[4-(phenylazo)-benzoyl]-glutamyl- $\gamma$-glutamic acid [PABGgG]) and inhibitor were made in Tris buffer (50 mM, pH 7.4). Working solutions of purified PSMA were diluted in Tris buffer $(50 \mathrm{mM}$, $\mathrm{pH} 7.4$, containing $1 \%$ Triton X-100 [Sigma-Aldrich]) to provide $15 \%-20 \%$ conversion of substrate to product in the absence of inhibitor. A typical incubation mixture (final volume, $250 \mu \mathrm{L}$ ) was prepared by the addition of either $25 \mu \mathrm{L}$ of an inhibitor solution or $25 \mu \mathrm{L}$ of Tris buffer (50 mM, pH 7.4) to $175 \mu \mathrm{L}$ of Tris buffer (50 mM, pH 7.4) in a test tube. PABGgG $(25 \mu \mathrm{L}, 10 \mu \mathrm{M})$ was added to the above solution. The enzymatic reaction was initiated by the addition of $25 \mu \mathrm{L}$ of the PSMA working solution. In all cases, the final concentration of PABGgG was $1 \mu \mathrm{M}$; the enzyme was incubated with 5 serially diluted inhibitor concentrations, providing a range of inhibition from $10 \%$ to $90 \%$. The reaction was allowed to proceed for $15 \mathrm{~min}$ with constant shaking at $37^{\circ} \mathrm{C}$ and was terminated by the addition of $25 \mu \mathrm{L}$ of methanolic trifluoroacetic acid ( $2 \%$ trifluoroacetic acid by volume in methanol), followed by vortexing. The quenched incubation mixture was quickly buffered by the addition of $25 \mu \mathrm{L}$ of $\mathrm{K}_{2} \mathrm{HPO}_{4}(0.1 \mathrm{M})$, vortexed, and centrifuged $(10 \mathrm{~min}$ at $7,000 \mathrm{~g})$. An $85-\mu \mathrm{L}$ aliquot of the resulting supernatant was subsequently quantified by HPLC as previously described $(18,19)$. $\mathrm{IC}_{50}$ values were calculated using KaleidaGraph 3.6 (Synergy Software).

\section{In Vivo PET Studies}

All animal experiments were conducted in accordance with the UCSF Institutional Animal Care and Use Committee guidelines. The ${ }^{18}$ F-fluorobenzamido-phosphoramidate (3) was evaluated in vivo using murine xenografts. Prostate cancer cell lines LNCaP (PSMA+, in 50/50 serum-free medium and Matrigel (BD Biosciences) matrix) and PC-3 (PSMA-negative, in 100\% serum-free medium) were used to inoculate the animals. Approximately $10^{7}$ LNCaP $(n=6)$ or PC-3 $(n=3)$ cells were injected subcutaneously on the right shoulder of male NCr nude mice. Animals with tumors 


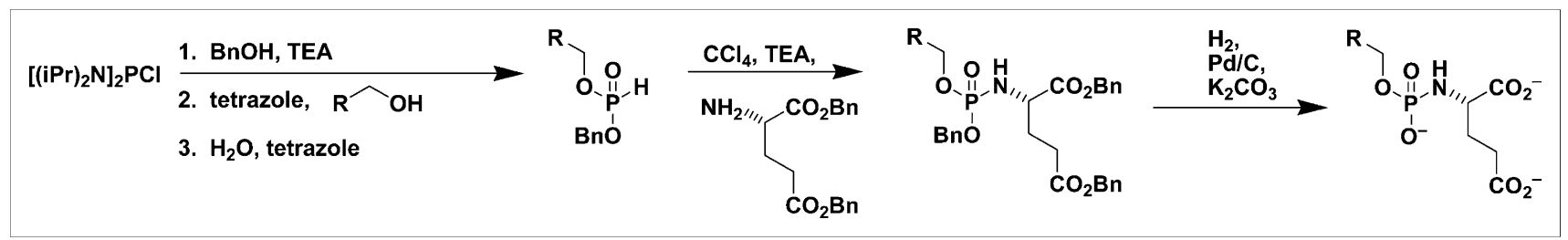

FIGURE 2. General scheme of modular synthetic approach to phosphoramidates.

measuring between 5 and $10 \mathrm{~mm}$ (1-2 wk after injection) were anesthetized by isoflurane inhalation. The synthesized ${ }^{18} \mathrm{~F}$-fluorobenzamido-phosphoramidate (3) $\quad(3,700-7,400 \quad \mathrm{kBq} \quad[100-200$ $\mu \mathrm{Ci}]$ ) was administered through tail vein injection. The animals were imaged by a microPET/CT system (Inveon; Siemens) at 0,1 , and $2 \mathrm{~h}$ for 10-min acquisition times. For blocking studies, animals were injected with $1 \mathrm{mg}$ of nonradioactive fluorobenzamidophosphoramidate (2) in $200 \mu \mathrm{L}$ of Tris buffer $1 \mathrm{~h}$ before injection of the radioactive tracer.

The PET data were acquired in list mode and reconstructed with the iterative ordered-subset expectation maximization 2-dimensional reconstruction algorithm provided with the Siemens Inveon System.

\section{Biodistribution Studies}

After imaging at $2 \mathrm{~h}$, animals were euthanized for biodistribution analysis. Blood was collected by cardiac puncture. Major organs-heart, lung, liver, pancreas, spleen, kidney, brain, and testes-and tumor xenografts were harvested, weighed, and counted in an automated $\gamma$-counter (Wizard 2; PerkinElmer). The percentage injected dose per gram $(\% \mathrm{ID} / \mathrm{g})$ of tissue was calculated by comparison with standards of known radioactivity.

Statistical analysis was performed using a $t$ test (Microsoft Excel software). All analyses were 1 tailed and considered a type 3 (2-sample unequal variance). A $P$ value of less than 0.05 was considered statistically significant.

\section{RESULTS}

Synthesis and Characterization of Phosphoramidate (1) and Its Conjugates

Using bis-(diisopropylamino) chlorophosphine (Cl-P$\left.\left[\mathrm{N}(i \mathrm{Pr})_{2}\right]_{2}\right)$ and protected glutamate, we have been able to generate the inhibitor core routinely. On the basis of this modular synthetic approach, a variety of primary alcohols have been incorporated with ease (Fig. 2). As previously described, systematic molecular pruning and computational chemistry have suggested that serine and glutamate at P1 and $\mathrm{P}^{\prime}$ positions are responsible for interacting with the PSMA $\mathrm{Arg}^{536}$ and $\mathrm{Arg}^{210} / \mathrm{Tyr}^{700} / \mathrm{Tyr}^{227}$ binding pockets, respectively (12). Further cell studies have led to the discovery of the leading phosphoramidate (1) with potent PSMA inhibition ( $\left.\mathrm{IC}_{50}, 14 \mathrm{nM}\right)(14)$. Following Figure 2, phosphoramidate (1) was synthesized at close to $90 \%$ yield. By incorporating the glutamate-serine dipeptide as a primary alcohol, this leading inhibitor also possesses an amine functional linker for incorporation of ${ }^{18} \mathrm{~F}$-labeled prosthetic groups for in vivo PET (Fig. 3).

To investigate the effect of the prosthetic group on the inhibitory potency of phosphoramidate (1), we opted to synthesize the nonradioactive analog of the fluorobenzamido-phosphoramidate (2) conjugate. Using standard bioconjugation techniques (20), we reacted a 3-fold excess of SFB with phosphoramidate (1) in buffered conditions. For the ease of separation, resin-bound isothiocyanate was used as a scavenger for unreacted phosphoramidate (1). Unreacted or hydrolyzed SFB was removed by successively triturating (2) with DMSO before subsequent inhibition studies.

Using the same conjugation chemistry through an $N$-succinimidyl ester, we efficiently coupled ${ }^{18} \mathrm{~F}-\mathrm{SFB}$ to phosphoramidate (1) in $10 \mathrm{~min}$, producing the radiolabeled PSMA inhibitor (3) at greater than $90 \%$ radiochemical yield. With the nonradioactive fluorobenzamido-phosphoramidate (2) as a standard, we have optimized HPLC conditions using a Fusion RP column (Phenomenex, Inc.) to purify the ${ }^{18}$ F-labeled product (3). Six different batches of the labeled product have been generated with consistent reproducibility.

\section{PSMA Inhibition}

The effect of SFB conjugation on PSMA inhibition by the phosphoramidate was investigated using an HPLCbased $\mathrm{IC}_{50}$ assay $(12,18,19)$. The nonradioactive fluorobenzamido conjugate (2) exhibited a higher inhibitory potency than did the parent phosphoramidate with the $\mathrm{IC}_{50}$ value of

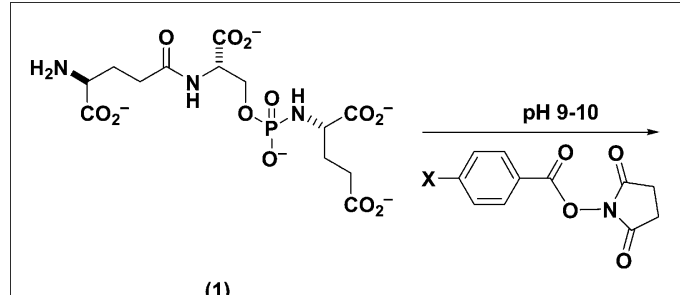

(1)

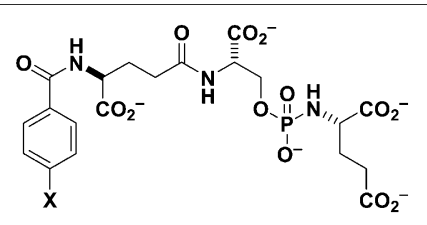

$X=F(2)$ or ${ }^{18} F(3)$
FIGURE 3. Synthetic scheme for 4fluorobenzamido-phosphoramidate conjugate (2) and 4-18F-fluorobenzamido-phosphoramidate conjugate (3). 

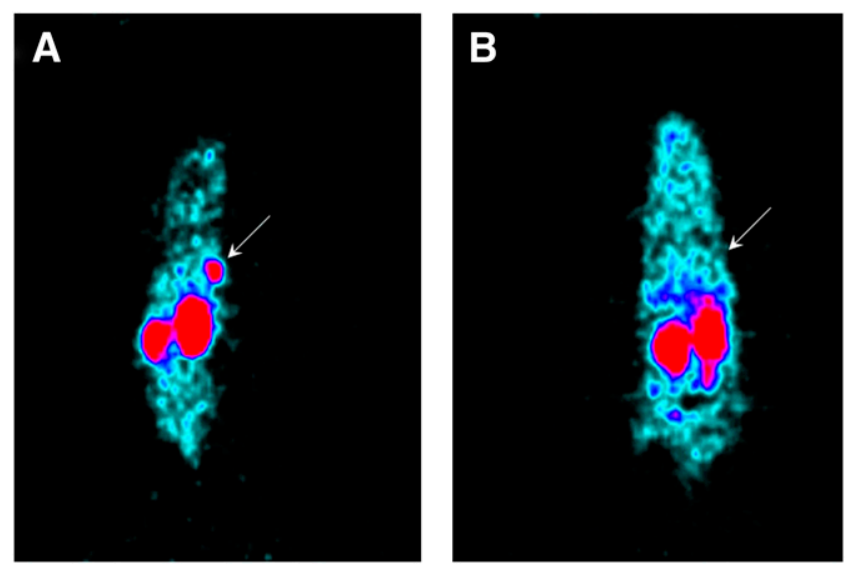

FIGURE 4. PET coronal images of male nude mice bearing subcutaneous LNCaP (A) and PC-3 (B) tumor xenografts $2 \mathrm{~h}$ after injection of ${ }^{18} \mathrm{~F}$-fluorobenzamido-phosphoramidate (3). Arrows indicate tumor placement.

$0.68 \mathrm{nM}$. A similar trend was also observed for the 5FAMXphosphoramidate conjugate (14), indicating that subsequent tagging of an imaging reporter does not adversely affect the inhibitory property of the leading phosphoramidate (1).

\section{In Vivo Imaging and Biodistribution Study}

${ }^{18} \mathrm{~F}$-fluorobenzamido-phosphoramidate (3) was injected via a tail vein into male $\mathrm{NCr}$ nude mice bearing $\mathrm{LNCaP}$ PSMA + or PC-3 PSMA - tumor xenografts. As shown in Figures 4 and 5, the in vivo uptake of (3) can clearly be observed in the LNCaP PSMA+ model at $2 \mathrm{~h}$ postinjection. In contrast, there was no detectable tumor signal in the PC-3 PSMA - xenograft. In both models, there is a significant uptake in kidneys but a modest degree of signal found in all other organs. The specificity of ${ }^{18} \mathrm{~F}-$ fluorobenzamido-phosphoramidate (3) to PSMA was demonstrated by the competition study with the blocking agent. The LNCaP PSMA+ model was injected with $1 \mathrm{mg}$ of the nonradioactive version of fluorobenzamido-phosphoramidate (2) an hour before the administration of ${ }^{18} \mathrm{~F}$-fluorobenzamido-phosphoramidate (3). The resulting PET images showed a substantial decrease in tumor uptake at $120 \mathrm{~min}$ after injection of the imaging probe (Fig. 5). A reduction in kidney uptake was also observed in the animals in the blocking experiment.

The ex vivo biodistribution data confirm the imaging findings (Fig. 6). The averaged tumor uptake in the PSMA+ model is 4 times higher than that of the PSMA- control. At $2 \mathrm{~h}$ post-injection, the PSMA+ tumor accumulation was $1.24 \pm 0.17 \% \mathrm{ID} / \mathrm{g}$, with a tumor-to-blood ratio of 9:1 (Fig. 7). With the exception of the kidneys, there was minimal nonspecific uptake in all other organs $(<0.25$ $\% \mathrm{ID} / \mathrm{g})$. Tumor uptake in the PSMA- control was comparable to the uptake in the normal organs $(0.32 \pm 0.14$ $\% \mathrm{ID} / \mathrm{g}$ ), with a tumor-to-blood ratio of $1: 1$. The differences of tumor uptake collected in the PSMA+ and PSMAanimal models were statistically significant, as confirmed by a 1 -tailed Student $t$ test with the $P$ value less than 0.002 . When animals were treated in advance with the nonradioactive fluorobenzamido-phosphoramidate (2), the uptake by the LNCaP PSMA+ tumor was decreased by 8 -fold $(0.13 \% \pm 0.14)$, with a tumor-to-blood ratio of $0.8: 1$. The kidney uptake in both PSMA + and PSMA - models was relatively high at $2.24 \pm 0.6 \% \mathrm{ID} / \mathrm{g}$ and $2.83 \pm 0.9$ $\% \mathrm{ID} / \mathrm{g}$, respectively. However, a significant decrease in kidney uptake ( $>6$-fold) was observed in mice pretreated with the nonradioactive blocking agent.

\section{DISCUSSION}

\section{Design of Phosphoramidates as PSMA Inhibitors}

Phosphoramidates, first described by Maung et al. (19), are potent PSMA inhibitors. The design strategy of this class of compounds is largely based on the binding features of PSMA endogenous substrates and potent inhibitors. As shown in Figure 1, the phosphoramidate scaffold is incorporated with L-glutamate at the $\mathrm{P}^{\prime}$ ' position, possessing a binding feature closely resembling L-glutamate in the folyl- $\gamma$-glu substrate. Compared with 2-PMPA, a known PSMA potent inhibitor, and the urea-based PSMA target agents (Fig. 1), phosphoramidates not only have similar structural features but also are well suited for carrying amine-reactive payloads while retaining excellent inhibitory potency.

In the past, a variety of phosphoramidates have been synthesized using a modular approach $(12,14)$ from Cl-P$\left[\mathrm{N}(i \mathrm{Pr})_{2}\right]_{2}$, protected glutamate, and primary alcohols. To closely mimic a PSMA substrate, we introduced the glutamate-serine dipeptide as a primary alcohol building block to complete the synthesis of phosphoramidate (1). In this particular compound, whereas the serine residue occupies the P1 position to provide an additional binding feature to the $\operatorname{Arg}^{536}$ pocket (12), the glutamate residue serves as a linker with amine functionality for convenient coupling of reporter molecules. Taken together, the overall design of phosphoramidate (1) possesses key functionalities

FIGURE 5. PET transaxial images of male nude mice bearing subcutaneous $\mathrm{LNCaP}$ (A and C) and PC-3 (B) tumor xenografts at $2 \mathrm{~h}$ after injection of ${ }^{18} \mathrm{~F}$ fluorobenzamido-phosphoramidate (3). Arrows indicate tumor placement. Blocked $\mathrm{LNCaP}$ is shown in $\mathrm{C}$.
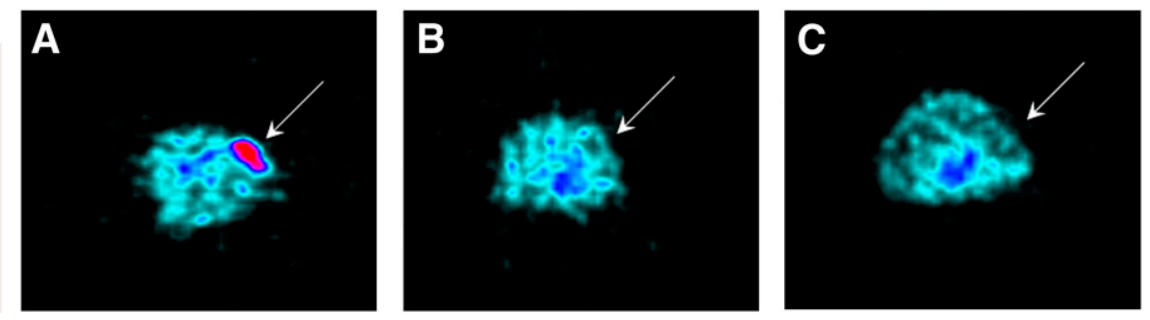


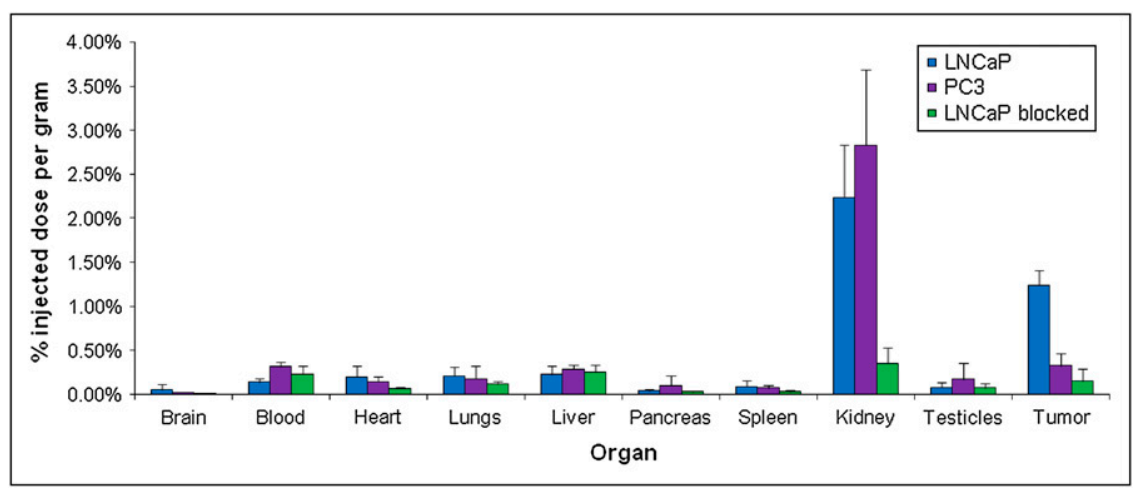

FIGURE 6. Biodistribution of ${ }^{18} \mathrm{~F}$-fluorobenzamido-phosphoramidate (3) as determined by radioactivity assays in tumor-bearing mice ( $n=3$ in each group). Tissues were harvested at $2 \mathrm{~h}$ after injection of ${ }^{18} \mathrm{~F}$-fluorobenzamidophosphoramidate (3). Uptake values are expressed as \%ID/g of tissue. for PSMA targeting and inhibition. With the established modular synthetic approach, phosphoramidate (1) can be routinely produced in high yield.

Phosphoramidate (1) itself is a potent PSMA inhibitor ( $\mathrm{IC}_{50}, 14 \mathrm{nM}$ ), exhibiting pseudo-irreversible inhibition that is common to this structural framework (13). Interestingly, the fluorobenzamido conjugate exhibits an enhanced inhibitory potency by 20 -fold. This trend is consistent with other conjugates such as 5FAMX (14) and presumably arises through the neutralization of the N-terminal amine through conjugation. Unlike some of the urea-based inhibitors (21), the glutamate residue also acts as a spacer for incorporation of reporter molecules, without causing any adverse effects on the intrinsic PSMA inhibition. This gives phosphoramidate (1) the flexibility to be used as a targeting ligand for different imaging strategies.

\section{In Vivo Imaging and Biodistribution}

Encouraged by the in vitro inhibition data (14), we examined the in vivo uptake of ${ }^{18} \mathrm{~F}$-fluorobenzamidophosphoramidate (3) in PSMA + and PSMA - tumor models by PET and ex vivo biodistribution study. Our initial assessment shows that the phosphoramidate probe (3) rapidly localized at the LNCaP PSMA + tumor, whereas there was significantly less activity found in the PC-3 PSMA - tumor. The specificity of the probe was tested using fluorobenzamido-phosphoramidate (2) in a competition study. When PSMA + animals were pretreated with $1 \mathrm{mg}$ of the nonradioactive analog of phosphoramidate (3) to block the binding pockets of PSMA, the resulting tumor uptake was significantly decreased. Such in vivo findings clearly demonstrate that the leading phosphoramidate is a PSMAspecific targeting ligand.

In addition to high PSMA + tumor uptake, the phosphoramidate probe also exhibits low background and negligible nonspecific binding to most organs. In particular, there is minimal uptake in bone, suggesting that no metabolic defluorination or hydrolysis of phosphoramidate to phosphate causing deposits in the bone hydroxyapatite matrix. The strong kidney uptake may in part be due to the hydrophilic nature of the probe, which favors renal clearance. More important, this may due to the strong PSMA expression found in mice that is also noted by others in the literature (22-24). This is further supported by the reduction of kidney signal by 6-fold when PSMA+ animals were treated in advance with the nonradioactive fluorobenzamido-phosphoramidate in the blocking study. Collectively, the strong uptake in the kidneys may be confined to the mouse model. Nonetheless, the mouse xenografts still serve as a good model for the initial evaluation for our probe development.

Biodistribution data confirm our findings from PET. At $120 \mathrm{~min}$, the phosphoramidate probe achieved tumor uptake of $1.24 \% \mathrm{ID} / \mathrm{g}$ in the LNCaP PMSA+ model, with a tumor-to-blood ratio of 9:1, which is significantly higher than the tumor-to-background criteria set by the Food and Drug Administration-approved agent ProstaScint (3:1) (25). Equally important, our data show that the phosphoramidate probe is specific to PSMA + tumors, with averaged PSMA+ tumor uptake 4 times higher than the PC-3 PSMA - model $(0.32 \% \mathrm{ID} / \mathrm{g})$. The PSMA specificity is further supported by the blocking study using a pretreatment of the nonradioactive probe $(1 \mathrm{mg})$, with the resulting tumor uptake reduced by 8-fold. Compared with a previously published ${ }^{18} \mathrm{~F}$-labeled urea-based agent (6),

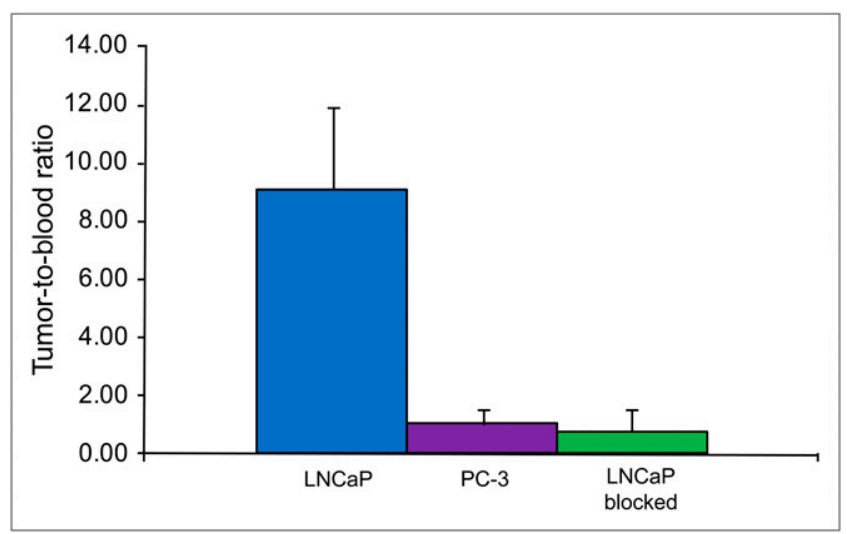

FIGURE 7. Tumor-to-blood ratios of male nude mice bearing subcutaneous LNCaP and PC-3 tumor xenografts $2 \mathrm{~h}$ after injection of ${ }^{18} \mathrm{~F}$-fluorobenzamido-phosphoramidate (3). LNCaP blocked indicates injection in advance of $1 \mathrm{mg}$ of nonradioactive fluorobenzamido-phosphoramidate (2). 
our phosphoramidate probe in the current form may have lower tumor uptake $(1.24 \% \mathrm{ID} / \mathrm{g}$ vs. $3.7 \% \mathrm{ID} / \mathrm{g})$ at $2 \mathrm{~h}$ after injection; however, our probe exhibits significantly lower kidney and liver uptake (0.21-0.23\% ID/g vs. 12-29\% ID/g) and a comparable tumor-to-blood ratio of 9:1.

\section{CONCLUSION}

Phosphoramidate (1) is a potent PSMA inhibitor with structural elements that closely resemble PSMA substrates. With L-glutamate as a spacer, the phosphoramidate scaffold possesses the flexibility to accommodate a variety of reporter molecules without compromising the PSMA inhibition properties, making it a unique platform for PSMA targeting. As an imaging agent, the ${ }^{18} \mathrm{~F}$-labeled phosphoramidate has high uptake in the PSMA-expressing tumor and low background. The in vivo data also show that the tumor uptake is specific to PSMA. These collectively positive findings provide confidence that further development of this class of compounds will yield a highly sensitive and specific imaging agent for prostate cancer.

\section{ACKNOWLEDGMENTS}

We thank Joseph K. Choi for expert assistance with $\mathrm{IC}_{50}$ determinations, Dr. Youngho Seo for his helpful discussions and advice, and William Mannone for reliable operation of the UCSF cyclotron. This work was supported by the National Cancer Institute (R21CA122126).

\section{REFERENCES}

1. Jemal A, Siegel R, Ward E, et al. Cancer statistics, 2008. CA Cancer J Clin. 2008;58:71-96.

2. Speight JL, Roach M, III. Advances in the treatment of localized prostate cancer: the role of anatomic and functional imaging in men managed with radiotherapy. J Clin Oncol. 2007;25:987-995.

3. Ghosh A, Heston WD. Tumor target prostate specific membrane antigen (PSMA) and its regulation in prostate cancer. J Cell Biochem. 2004;91: 528-539.

4. Tricoli JV, Schoenfeldt M, Conley BA. Detection of prostate cancer and predicting progression: current and future diagnostic markers. Clin Cancer Res. 2004;10:3943-3953.

5. Foss CA, Mease RC, Fan H, et al. Radiolabeled small-molecule ligands for prostate-specific membrane antigen: in vivo imaging in experimental models of prostate cancer. Clin Cancer Res. 2005;11:4022-4028.

6. Chen Y, Foss CA, Byun Y, et al. Radiohalogenated prostate-specific membrane antigen (PSMA)-based ureas as imaging agents for prostate cancer. J Med Chem. 2008;51:7933-7943.
7. Mease RC, Dusich CL, Foss CA, et al. $N$-[N-[(S)-1,3-dicarboxypropyl]carbamoyl]-4-[ $\left.{ }^{18} \mathrm{~F}\right]$ fluorobenzyl-L-cysteine, $\left[{ }^{18} \mathrm{~F}\right] \mathrm{DCFBC}$ : a new imaging probe for prostate cancer. Clin Cancer Res. 2008;14:3036-3043.

8. Pinto JT, Suffoletto BP, Berzin TM, et al. Prostate-specific membrane antigen: a novel folate hydrolase in human prostatic carcinoma cells. Clin Cancer Res. 1996;2:1445-1451.

9. Heston WD. Characterization and glutamyl preferring carboxypeptidase function of prostate specific membrane antigen: a novel folate hydrolase. Urology. 1997;49:104-112.

10. Barinka C, Rinnova M, Sacha P, et al. Substrate specificity, inhibition and enzymological analysis of recombinant human glutamate carboxypeptidase II. J Neurochem. 2002;80:477-487.

11. Mallari JP, Choy CJ, Hu Y, et al. Stereoselective inhibition of glutamate carboxypeptidase by organophosphorus derivatives of glutamic acid. Bioorg Med Chem. 2004;12:6011-6020.

12. Wu LY, Anderson MO, Toriyabe Y, et al. The molecular pruning of a phosphoramidate peptidomimetic inhibitor of prostate-specific membrane antigen. Bioorg Med Chem. 2007;15:7434-7443.

13. Liu T, Toriyabe Y, Kazak M, Berkman CE. Pseudoirreversible inhibition of prostate-specific membrane antigen by phosphoramidate peptidomimetics. Biochemistry. 2008.

14. Liu T, Wu LY, Kazak M, Berkman CE. Cell-surface labeling and internalization by a fluorescent inhibitor of prostate-specific membrane antigen. Prostate. 2008;68:955-964.

15. Wust F, Hultsch C, Bergmann R, Johannsen B, Henle T. Radiolabelling of isopeptide $\mathrm{N}$ epsilon-( $\gamma$-glutamyl)-L-lysine by conjugation with $\mathrm{N}$-succinimidyl4- $\left[{ }^{18} \mathrm{~F}\right]$ fluorobenzoate. Appl Radiat Isot. 2003;59:43-48.

16. Lapatsanis L, Milas G, Froussios K, Kolovos M. N-2,2,2-(trichloroethoxycarbonyl)-L-amino acids and synthesis of $N$-(9-fluoroenymethoxycarbonyl)-L-amino acids involving succininidoxy anion as a leaving group in amino acid protection. Synthesis. 1983;8:671-673.

17. Mading P, Fuchtner F, Wust F. Module-assisted synthesis of the bifunctional labelling agent $\mathrm{N}$-succinimidyl $4-\left[{ }^{18} \mathrm{~F}\right]$ fluorobenzoate $\left(\left[{ }^{18} \mathrm{~F}\right] \mathrm{SFB}\right)$. Appl Radiat Isot. 2005;63:329-332.

18. Anderson MO, Wu LY, Santiago NM, et al. Substrate specificity of prostatespecific membrane antigen. Bioorg Med Chem. 2007;15:6678-6686.

19. Maung J, Mallari JP, Girtsman TA, et al. Probing for a hydrophobic a binding register in prostate-specific membrane antigen with phenylalkylphosphonamidates. Bioorg Med Chem. 2004;12:4969-4979.

20. Hermanson GT. Bioconjugation Techniques. San Diego, CA: Academic Press, Inc.; 1996.

21. Banerjee SR, Foss CA, Castanares M, et al. Synthesis and evaluation of technetium-99m- and rhenium-labeled inhibitors of the prostate-specific membrane antigen (PSMA). J Med Chem. 2008;51:4504-4517.

22. Aggarwal S, Ricklis RM, Williams SA, Denmeade SR. Comparative study of PSMA expression in the prostate of mouse, dog, monkey, and human. Prostate. 2006;66:903-910.

23. Gregor PD, Wolchok JD, Turaga V, et al. Induction of autoantibodies to syngeneic prostate-specific membrane antigen by xenogeneic vaccination. Int $J$ Cancer. 2005; 116:415-421.

24. Klein EA. Management of Prostate Cancer. 2nd ed. Totowa, NJ: Humana Press; 2004.

25. Sodee DB, Ellis RJ, Samuels MA, et al. Prostate cancer and prostate bed SPECT imaging with ProstaScint: semiquantitative correlation with prostatic biopsy results. Prostate. 1998;37:140-148. 